\title{
PLANTA EXPERIMENTAL PARA SISTEMAS DE CALEFACCIÓN DE VEHÍCULOS DE TRANSPORTE PÚBLICO
}

\author{
María L. Delgado, Manuel Lara, Francisco Vázquez \\ Departamento de Ingeniería Eléctrica y Automática, Universidad de Córdoba, Campus de Rabanales, \\ Edificio Leonardo Da Vinci, Córdoba, España, p72degum@uco.es
}

\begin{abstract}
Resumen
En este trabajo se describe una planta experimental para test de sistemas de calefacción de vehículos de transporte público. La planta incorpora un sistema para el control de la temperatura de la cabina $y$ emula la temperatura del motor del vehículo (mediante una caldera de gas natural), así como la temperatura exterior del autobús. De este modo, es posible imponer diferentes condiciones de operación al ciclo de calefacción. Se expone además la estrategia de control utilizada y, como ejemplo, los datos obtenidos en un ensayo realizado con la planta, que junto con otras experiencias servirán para la caracterización de sus componentes. Con este trabajo se dispone de una planta experimental que servirá como banco de pruebas para explorar distintas estrategias de control y optimización orientadas a aumentar el rendimiento de los sistemas de calefacción de vehículos de transporte público.
\end{abstract}

Palabras clave: Sistema de calefacción, Transporte público, Control.

\section{INTRODUCCIÓN}

Una de las principales preocupaciones de la industria del automóvil es el confort de sus pasajeros, sobre todo en los vehículos destinados al transporte público. Por tanto, el diseño, optimización y control de los sistemas de calefacción, ventilación y aire acondicionado (HVAC, Heating, Ventilating and Air Conditioning) de vehículos para mantener el confort térmico de sus pasajeros es un problema técnico relevante. Existen diferentes trabajos que tratan está temática, algunos orientados a obtener modelos térmicos de la cabina del vehículo [5], [3], otros enfocados en el confort térmico de los pasajeros teniendo en cuenta condiciones de temperatura, humedad y velocidad del aire [6] o centrados en estudiar el rendimiento de cada componente del sistema de aire acondicionado [10].

La optimización y el control de los sistemas HVAC [7] es más relevante en los vehículos destinados al uso público y en concreto, en los autobuses debido a ciertos factores particulares de estos automóviles como son su bajo aislamiento debido a la reducción de peso necesaria en la propia carrocería, la ocupación variable [4], las perturbaciones producidas por las frecuentes aperturas de las puertas, o las grandes no linealidades tanto en el comportamiento del intercambiador de calor como en la válvula de control [8]. Todas estas características dificultan el mantenimiento del confort térmico del autobús, y se añaden a ciertas condiciones de operación, como es el caso de la deshumidificación en la que se prima la seguridad (visión del conductor) al confort.

Otra característica importante de estos sistemas es el carácter multivariable del proceso. La mayoría de los autobuses, y muchos otros vehículos, disponen de un doble sistema de climatización, estableciendo dos zonas [9], la cabina del conductor y la zona de pasajeros, cada una con su consigna de temperatura. Dado que la zona de pasajeros tiene unas dimensiones considerablemente mayores que la zona de conductor, esta última se ve condicionada por la primera, produciéndose acoplamientos entre los dos lazos de control.

Por otra parte, y considerando en específico los sistemas de calefacción de los autobuses, la energía procede del sistema de refrigeración del motor de combustión. Pero esta energía no es constante ni en los arranques ni durante el funcionamiento normal, en el que el motor está sometido a los cambios propios del régimen del vehículo (variaciones de velocidad, pendientes, demanda por parte de otros sistemas, etc.), limitando su disponibilidad. $\mathrm{Al}$ mismo tiempo, las restricciones impuestas a los motores de combustión con la entrada en vigor de la normativa europea EURO VI y posteriores, limitan el paso de agua caliente al circuito de calefacción mientras no se alcanza la temperatura óptima de funcionamiento del motor, haciendo necesario el uso de precalentadores. Además, actualmente, el mercado tiende hacia los vehículos híbridos o totalmente eléctricos, en los que no existe como fuente de calor el motor de combustión, sino que dependen de una batería, lo que dificulta el mantenimiento del confort térmico dentro del habitáculo del vehículo. El hecho de emplear dicha batería para climatizar implica disminuir considerablemente la autonomía del vehículo [1],[2]. Por estas razones, actualmente se 
buscan alternativas al sistema de calefacción o su optimización, considerándose tanto la gestión de componentes (válvulas, bombas, etc.) como la mejora de eficiencia de los intercambiadores.

Con el objetivo de contribuir al conocimiento de los sistemas de calefacción, a la optimización de sus condiciones de operación y de explorar distintas estrategias de control para aumentar el rendimiento de los intercambiadores de calor, así como del resto de componentes que conforman el sistema, se ha diseñado una planta experimental con los principales elementos de este tipo de sistemas, parte de ellos cedidos por la empresa colaboradora, incluyendo una Unidad Central de Climatización (UCC) de un autobús real.

El resto del artículo se organiza de este modo: en la sección 2 se describe la planta experimental diseñada, en la sección 3 se detalla el sistema de control implementado, en la sección 4 se exponen los resultados obtenidos en un ensayo experimental realizado con esta planta que servirán de base para modelar sus componentes principales y, por último, en la sección 5 se resumen las conclusiones obtenidas y se especifican las líneas futuras del proyecto.

\section{DESCRIPCIÓN DE LA PLANTA}

La planta experimental diseñada, que se muestra en la Figura 1, consta principalmente de la UCC y una bomba de impulsión de agua, ambas pertenecientes al circuito de climatización del conductor de un autobús real, y de un sistema para emular el agua caliente procedente del motor de combustión. Se ha añadido la instrumentación necesaria para monitorizar el comportamiento de las variables de interés, con sensores de temperatura, caudal y presión.

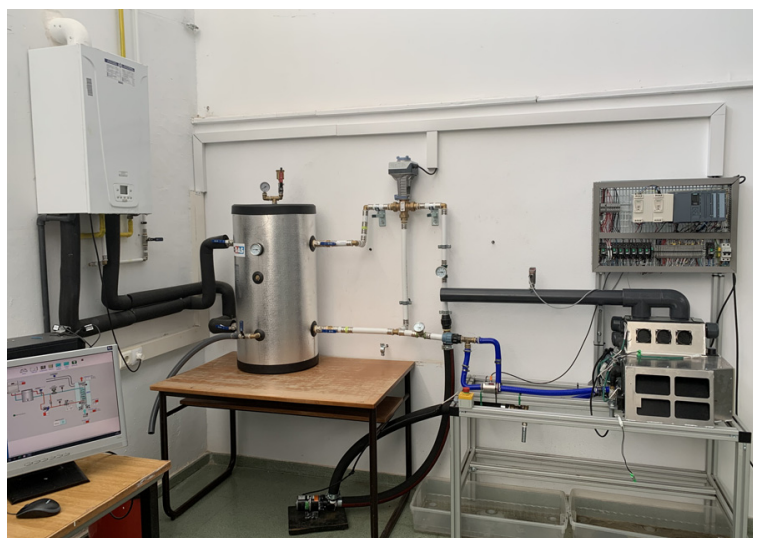

Figura 1: Planta experimental

Para la supervisión del estado de los sensores y el control de los actuadores, se ha empleado un sistema de control compuesto por un PLC (Programmable
Logic Controller) S7-1512 de Siemens, en el que se implementan las estrategias de control a bajo nivel, y un PC, en el que se ejecuta un SCADA (Supervisory Control And Data Acquisition), el WinCC de Siemens, así como el software Matlab (licencia Campus), para establecer estrategias de control de alto nivel (optimización, registro de datos, etc.).

En la Figura 2 aparecen los elementos principales que conforman la planta experimental, y que se describen en los siguientes apartados.

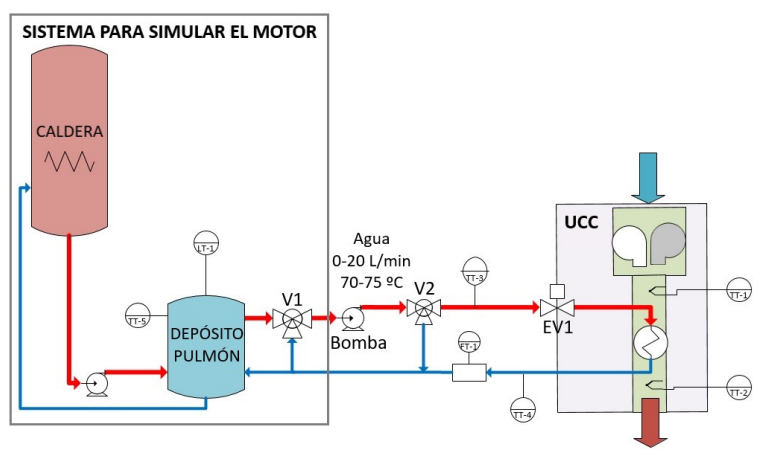

Figura 2: Esquema actual planta

\subsection{EMULACIÓN DEL MOTOR}

Para simular el calor producido por el motor de combustión de un autobús se ha empleado una caldera de gas natural de $24 \mathrm{~kW}$ de potencia calorífica y un depósito pulmón de 100 L. Además, se ha incorporado una válvula de tres vías de control proporcional de Siemens, V1, a la salida del depósito para reproducir el comportamiento del motor en los arranques, en los cuales existe un régimen transitorio hasta alcanzar la temperatura normal de funcionamiento. Esta válvula está pilotada por el PLC mediante una señal de 4-20 mA, con la que se modifica la posición del vástago, y a través de una entra analógica de $0-10 \mathrm{~V}$ se puede comprobar el feedback de la posición.

\subsection{IMPULSIÓN AGUA}

Para impulsar el agua caliente e introducirla en la UCC se emplea una bomba hidráulica que produce un caudal de $5000 \mathrm{~L} / \mathrm{h}$ (0,2 bar). Para su control se emplea una salida digital que activa y desactiva, a través de un relé, su alimentación ( $24 \mathrm{~V}$ DC). Por otra parte, Además, se ha incluido una válvula manual de tres vías, V2, para poder modificar el caudal máximo que entra en la UCC, haciendo que parte de este caudal de agua caliente no circule por la unidad de climatización, con la finalidad de probar su influencia sobre la temperatura de canalización de aire del autobús. 


\section{$2.3 \quad \mathrm{UCC}$}

El elemento principal de la planta es la UCC (Figura $3)$. Se compone esencialmente de un intercambiador de calor agua-aire, en el que se produce la transferencia de calor entre el agua caliente impulsada por la bomba procedente del circuito de refrigeración del motor y aire, que puede proceder del interior (recirculado) o del exterior del autobús. Esta transferencia se produce gracias a sus dos ventiladores que impulsan el aire y lo hacen circular de manera perpendicular a la batería del intercambiador por la que pasa el agua caliente. Para variar la velocidad de estos ventiladores se utiliza modulación por ancho de impulso (PWM, PulseWidth Modulation).

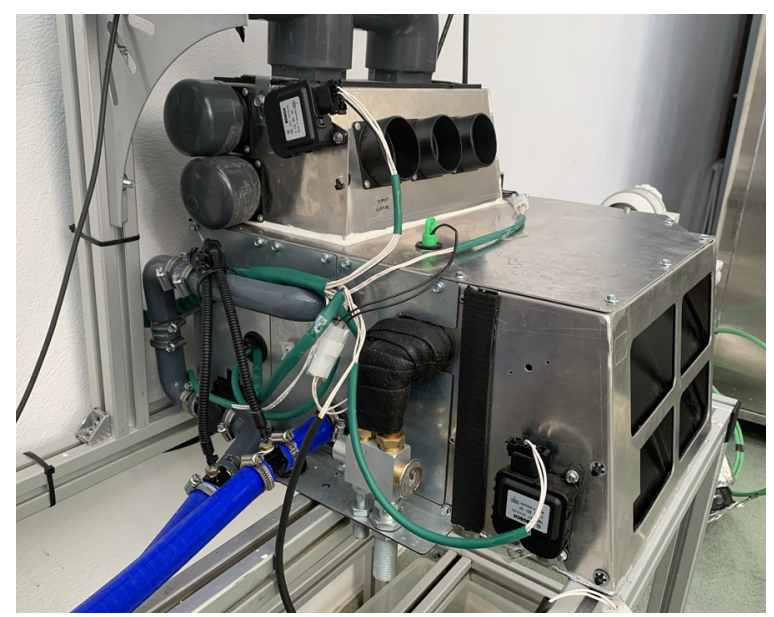

Figura 3: UCC

Otro elemento a destacar de esta unidad es la válvula de control, encargada de limitar el caudal de agua caliente a la entrada de la UCC. Esta válvula motorizada es gestionada por el PLC mediante dos salidas digitales que accionan el motor, una para la orden de marcha directa y otra para la orden de marcha inversa, modificando de esta forma la posición del obturador. Esta válvula posee un potenciómetro con el que se monitoriza la posición del vástago.

La UCC incorpora dos motores de corriente continua para modificar la posición de dos compuertas. Una de estas compuertas se emplea para seleccionar la procedencia del aire, del interior o del exterior del autobús. La otra, que incorpora un potenciómetro para poder establecer posiciones intermedias, se utiliza para la deshumidificación de la luna delantera del vehículo. Modificando la posición de esta compuerta, parte del caudal de aire caliente procedente del intercambiador se emplea en la deshumidificación en lugar de circular por las canalizaciones del sistema de calefacción.
La unidad de climatización incorpora tres sondas de temperatura, modelo KTY81/110, que se han conectado a tres entradas analógicas de $0-10 \mathrm{~V}$ del PLC mediante un divisor de tensión.

Puesto que para caracterizar el sistema se necesitan las temperaturas de los fluidos, así como sus caudales, se han añadido dos sondas de temperatura (KTY81/110) de agua tanto a la entrada como a la salida de la UCC, y dos caudalímetros, uno para medir el caudal de agua a la salida de la UCC, modelo SA6000 de IFM, y otro el de aire en la tobera de salida, que se corresponde con el caudal empleado para calentar la cabina del conductor, modelo SA5000 de IFM. Ambos caudalímetros se conectan al controlador, mediante dos entradas analógicas de 4-20 mA. Indicar que la tobera de salida no existe en el autobús real, sino que se ha construido y acoplado sobre la UCC para canalizar todo el aire que circula por la unidad en un único punto $\mathrm{y}$, de este modo, poder medir su caudal.

\subsection{EMULACIÓN DE LA TEMPERATURA AMBIENTE}

Se ha realizado una adaptación en la planta para poder reproducir condiciones ambientales difíciles de alcanzar en Córdoba. Para ello se conecta a la planta, una cámara de refrigeración capaz de alcanzar $0{ }^{\circ} \mathrm{C}$ de temperatura, y mediante una soplante, se extrae aire frío de su interior conduciéndolo hasta la entrada de la UCC como se muestra en la Figura 4. Para el control del extractor de aire de la cámara se ha empleado una salida digital del PLC que a través de un relé establece o corta su alimentación.

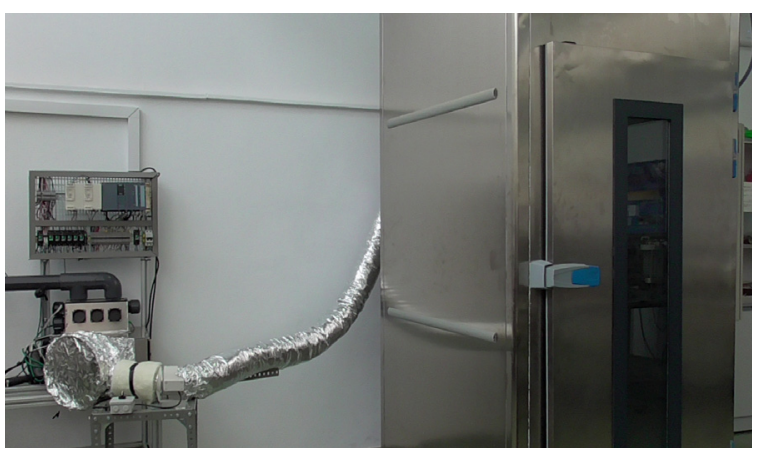

Figura 4: Adaptación planta

\subsection{SEÑALES}

En la Tabla 1 se especifican las señales de entrada y salida conectadas al PLC de la planta, indicando el tipo (AI, entrada analógica, AQ, salida analógica, DI, entrada digital o DQ, salida digital) y la unidad de medida, en su caso. 
Tabla 1: Señales conectadas al controlador

\begin{tabular}{|c|c|c|}
\hline Señal & Tipo & Unidad \\
\hline $\begin{array}{l}\text { Potenciómetro válvula } \\
\text { control de caudal (UCC) }\end{array}$ & AI & $\%$ \\
\hline $\begin{array}{l}\text { Potenciómetro válvula } \\
\text { compuerta luna (UCC) }\end{array}$ & AI & $\%$ \\
\hline Caudalímetro agua & AI & $1 / \mathrm{min}$ \\
\hline Caudalímetro aire & AI & $\mathrm{m}^{3} / \mathrm{h}$ \\
\hline $\begin{array}{l}\text { Consigna posición válvula } \\
\text { motorizada de } 3 \text { vías }\end{array}$ & AQ & $\%$ \\
\hline Activación ventiladores & DQ & - \\
\hline PWM ventiladores & $\mathrm{DQ}$ & - \\
\hline Bomba & DQ & - \\
\hline $\begin{array}{l}\text { Orden marcha (O.M.) } \\
\text { directa motor válvula control } \\
\text { de caudal (UCC) }\end{array}$ & DQ & - \\
\hline $\begin{array}{l}\text { O.M. inversa motor válvula } \\
\text { control de caudal (UCC) }\end{array}$ & DQ & - \\
\hline $\begin{array}{l}\text { O.M. directa motor válvula } \\
\text { compuerta pies (UCC) }\end{array}$ & DQ & - \\
\hline $\begin{array}{l}\text { O.M. inversa motor válvula } \\
\text { compuerta pies (UCC) }\end{array}$ & DQ & - \\
\hline Encendido caldera de gas & DQ & - \\
\hline $\begin{array}{l}\text { Activación soplante cámara } \\
\text { frigorífica }\end{array}$ & DQ & - \\
\hline $\begin{array}{l}\text { O.M. directa motor válvula } \\
\text { compuerta luna (UCC) }\end{array}$ & DQ & - \\
\hline $\begin{array}{l}\text { O.M. inversa motor válvula } \\
\text { compuerta luna (UCC) }\end{array}$ & DQ & - \\
\hline Seta de emergencia & DI & - \\
\hline $\begin{array}{l}\text { Temperatura aire de entrada } \\
\text { (UCC) }\end{array}$ & AI & ${ }^{\circ} \mathrm{C}$ \\
\hline $\begin{array}{l}\text { Temperatura aire exterior } \\
\text { (UCC) }\end{array}$ & AI & ${ }^{\circ} \mathrm{C}$ \\
\hline $\begin{array}{l}\text { Temperatura aire de salida } \\
\text { (UCC) }\end{array}$ & AI & ${ }^{\circ} \mathrm{C}$ \\
\hline $\begin{array}{l}\text { Temperatura agua de entrada } \\
\text { (UCC) }\end{array}$ & AI & ${ }^{\circ} \mathrm{C}$ \\
\hline $\begin{array}{l}\text { Temperatura agua de salida } \\
\text { (UCC) }\end{array}$ & AI & ${ }^{\circ} \mathrm{C}$ \\
\hline $\begin{array}{l}\text { Posición válvula motorizada } \\
\text { de } 3 \text { vías }\end{array}$ & AI & $\%$ \\
\hline
\end{tabular}

\section{SISTEMA DE CONTROL Y SUPERVISIÓN}

La planta está instrumentalizada con sensores de presión, temperatura y caudal. Para recoger los datos de estos sensores y controlar los actuadores, válvulas, motores y bomba, se ha empleado un sistema de control con una CPU 1512C-1 PN (Figura 5) que permite la implementación de redes Profinet y OPC UA (Open Platform Communications - Unified Architecture), así como modulación por ancho de pulso (PWM).

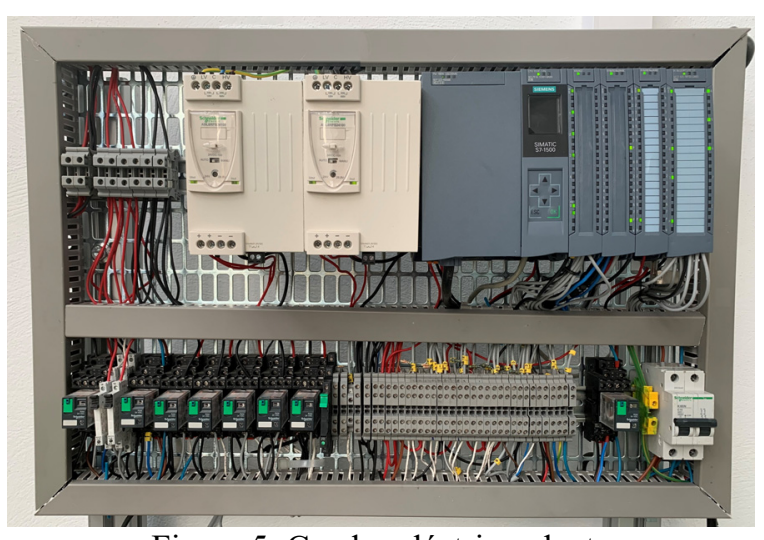

Figura 5: Cuadro eléctrico planta

\subsection{COMUNICACIÓN OPC UA}

Se ha configurado el PLC para que funcione como servidor OPC UA. Este protocolo, basado en Ethernet, es una evolución del clásico OPC, que permite realizar una comunicación, entre procesos o en red, independiente del fabricante y de la plataforma empleada con mecanismos de seguridad en el nivel de protocolo y de aplicación, entre otras ventajas. Se ha utilizado para comunicar variables entre el PLC y el software Matlab/Simulink que se ejecuta en un PC (Figura 6).

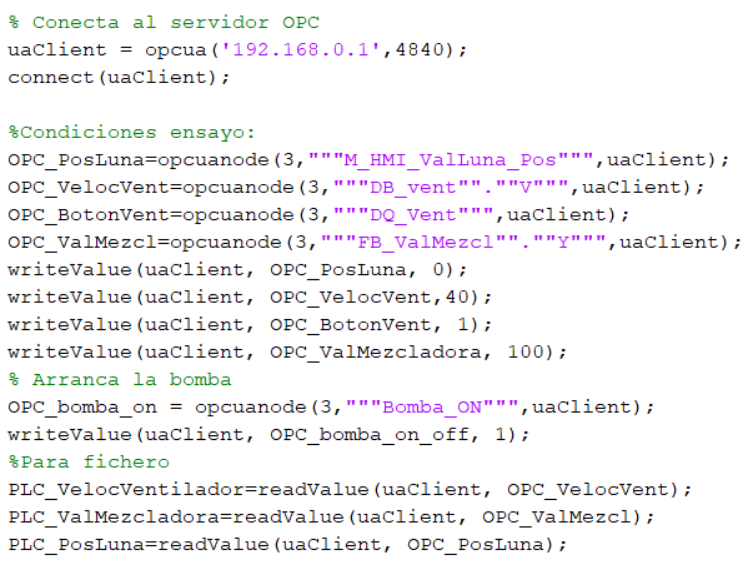

Figura 6: Captura código Matlab

\subsection{SCADA}

Para el control y supervisión de la planta experimental se ha diseñado un SCADA sobre PC, cuya pantalla principal se muestra en la Figura 7.

Desde este SCADA, además de la monitorización del estado sensores y el control de los actuadores de la planta desde la pantalla principal, se han incorporado otras funciones:

- calibración de las señales analógicas de entrada;

- configuración de los parámetros de los controladores PID; 
- almacenamiento de los datos generados en un fichero;

- gráficas con la variación temporal de las principales variables (Figura 8);

- visualización de la información del proyecto.

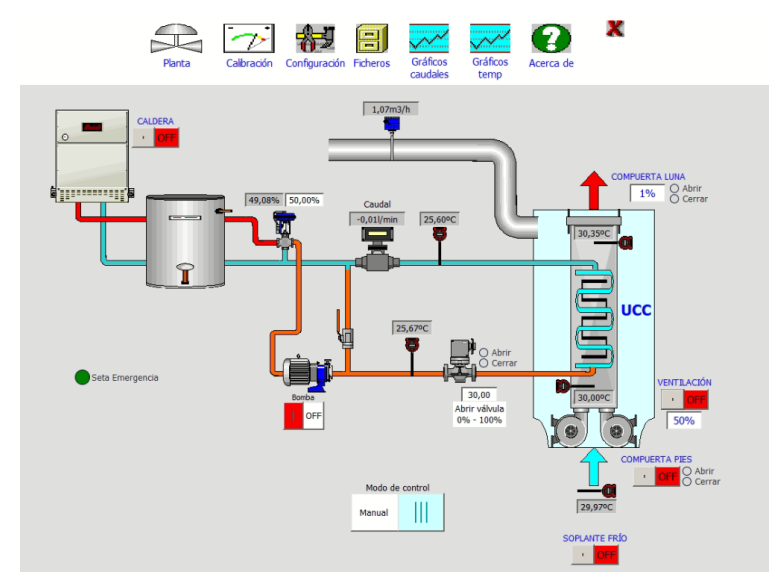

Figura 7: Pantalla principal SCADA

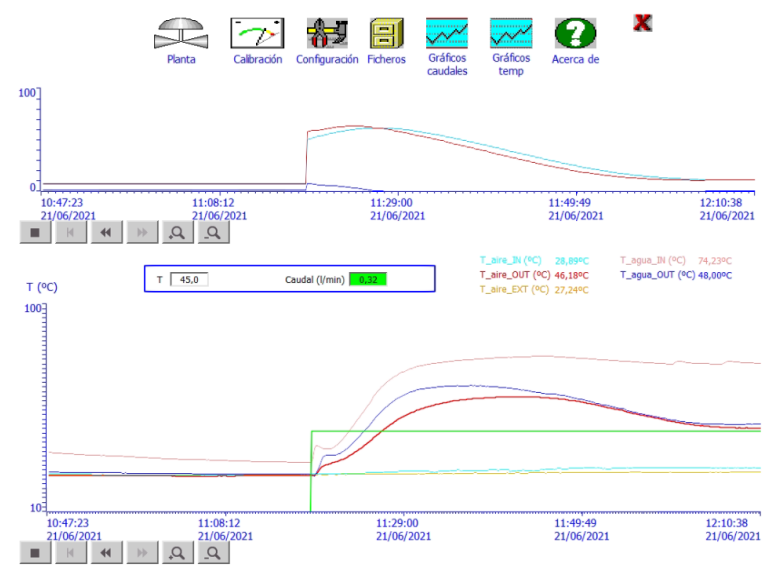

Figura 8: Gráficas temperaturas

\subsection{LAZOS DE CONTROL}

Desde el punto de vista de control, el objetivo principal es mantener controlada la temperatura de aire de salida, denominada de canalización, que se corresponde con la que es introducida en el recinto del conductor del autobús. La variable manipulada es el porcentaje de apertura de la válvula de la UCC. El resto de las señales se consideran perturbaciones. Muchas de estas perturbaciones se pueden reproducir de forma experimental en la planta.

Para el control de la temperatura del aire de canalización del circuito del conductor se ha empleado un controlador de tipo PID, aunque la acción derivativa no se ha utilizado. Para implementar este PID, en lugar de utilizar funciones predefinidas del PLC, se ha desarrollado un bloque propio para tener mayor flexibilidad. En este PID, se ha implementado el mecanismo anti-windup y el de histéresis y como periodo de muestreo se ha seleccionado $15 \mathrm{~s}$, tiempo similar al utilizado en la tarjeta de control de los autobuses. Para sintonizar este controlador se han empleado reglas heurísticas, basadas en la respuesta en lazo abierto en las que se han obtenido las relaciones entre el porcentaje de apertura de la válvula de control y el caudal, y entre el porcentaje de apertura de la válvula y la temperatura de aire de canalización.

\section{PRUEBAS EXPERIMENTALES}

A continuación, se presentan algunos datos obtenidos de un ensayo, a modo de ejemplo, realizado en la planta experimental con el objetivo de evaluar el control implementado.

En este ensayo en lazo cerrado se disminuyó la consigna de temperatura de canalización desde los 60 ${ }^{\circ} \mathrm{C}$ hasta $30{ }^{\circ} \mathrm{C}$, en decrementos de $5{ }^{\circ} \mathrm{C}$ cada $90 \mathrm{~min}$. La duración total del ensayo fue de $12 \mathrm{~h}$. La consigna de la caldera se mantuvo a $75{ }^{\circ} \mathrm{C}$, temperatura análoga a la de un motor de combustión de un autobús, y se programó la válvula mezcladora para que su apertura fuera lineal de $0-100 \%$ en $15 \mathrm{~min}$ reproduciendo el arranque del vehículo. Se limitó el caudal máximo de entrada a la UCC a $2 \mathrm{l} / \mathrm{min}$ empleando la válvula de tres vías manual, V2, y se seleccionó $2{ }^{\circ} \mathrm{C}$ como valor umbral para la histéresis del controlador.
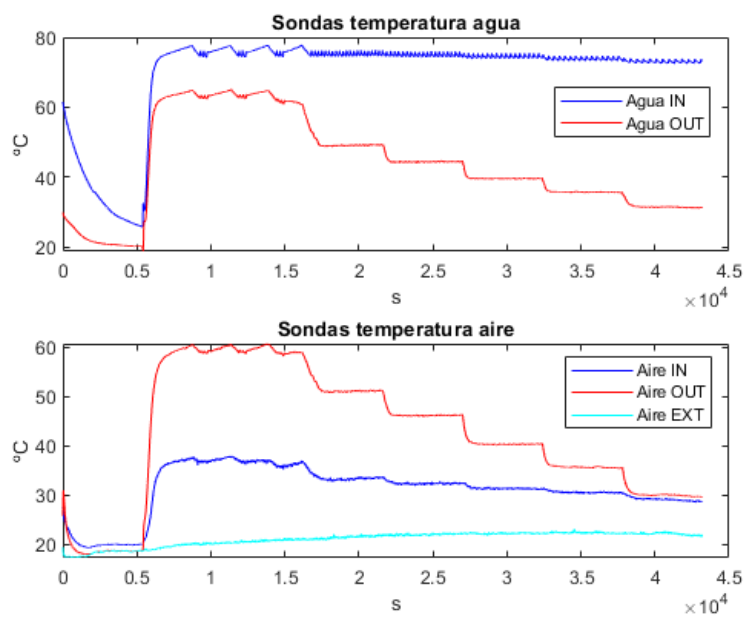

Figura 9: Sondas temperatura

En la Figura 9 se muestra la evolución temporal de las sondas de temperatura que caracterizan el sistema durante el ensayo en lazo cerrado. Se observa que la caldera es capaz de mantener la temperatura de entrada de la UCC prácticamente constante y 
alrededor de los $75{ }^{\circ} \mathrm{C}$, temperatura normal de funcionamiento del motor de combustión.

En la Figura 10, se representa, por un lado, la señal de control junto a la medida del potenciómetro de la válvula de control, ambas coincidentes, $\mathrm{y}$, por otro lado, la evolución de la temperatura de canalización del circuito de conductor junto a la referencia.
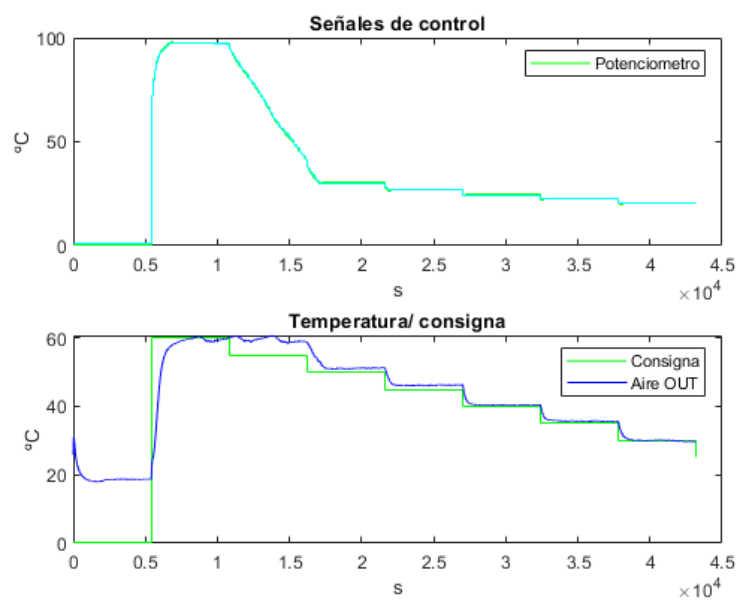

Figura 10: Respuesta y señal de control

En las gráficas anteriores, se puede observar el efecto del mecanismo de histéresis implementado en el PID, dejando constante la señal de control cuando la diferencia entre la temperatura de canalización y la consigna es inferior al valor umbral. Así mismo, se comprueba el seguimiento de referencias de la temperatura de canalización. La consigna de $55^{\circ} \mathrm{C}$ no se alcanza debido al tiempo que emplea el controlador en cerrar la válvula, que en el instante anterior al cambio de referencia se encuentra totalmente abierta. Sobre este fenómeno que se produce en el arranque de cualquier vehículo ya se han implementado soluciones, no descritas para preservar la confidencialidad.

\section{CONCLUSIONES Y TRABAJOS FUTUROS}

En este trabajo se ha descrito una planta de ensayos para sistemas de calefacción de vehículos públicos. La funcionalidad de la planta para emular la temperatura del agua del circuito de refrigeración del motor de combustión y la temperatura ambiente exterior del autobús, permite establecer diferentes condiciones de trabajo del sistema de calefacción. Además, se ha implementado un sistema de control y adquisición de datos sobre PC que posibilita el registro de todas las variables de interés de cada ensayo.
Como trabajos futuros, se emplearán los datos obtenidos en los ensayos para caracterizar y modelar los componentes de la planta, permitiendo de esta forma obtener un modelo térmico del autobús. Actualmente, se está trabajando en un modelo térmico híbrido del vehículo, en el que, por un lado, como parte simulada se emplea un modelo térmico en Matlab del habitáculo del autobús y del circuito de calefacción de la zona de pasajeros y, por otro lado, como parte real se utiliza la planta experimental descrita en este trabajo que emula el circuito de calefacción del conductor. Se prevé realizar una batería de ensayos empleando este modelo híbrido y comparar sus resultados con los obtenidos con el modelo térmico completo y con los datos recogidos en pruebas en autobuses reales. Por último, se implementarán estrategias de control y optimización avanzadas con el objetivo de aumentar el rendimiento de los sistemas de calefacción de vehículos de transporte público.

\section{Agradecimientos}

Agradecer a la Consejería de Transformación Económica, Industria, Conocimiento y Universidades de la Junta de Andalucía la financiación del proyecto P18-TP-2040, y a la empresa Hispacold (grupo Irizar), copartícipe del citado proyecto.

Por otro lado, agradecer también la contribución de los alumnos del grado de Ingeniería Electrónica Industrial participantes en el proyecto, como $\mathrm{M}^{\mathrm{a}}$ de los Reyes Mármol o Alfonso y Antonio Almenara, en diversas fases de su implementación.

\section{English summary}

\section{EXPERIMENTAL PLANT FOR HEATING SYSTEMS IN PUBLIC TRANSPORT VEHICLES}

\begin{abstract}
In this paper, an experimental plant for heating systems of public transport vehicles is described. The plant incorporates a system to control the cabin temperature and allows the emulation of the vehicle engine temperature as well as the outside temperature of the bus. In this way, different operating conditions on the heating cycle can be imposed. The control strategy employed is described and data obtained from a specific experimental setup are analyzed. This test-bench will be useful for comparing and studying different control optimization strategies aimed at increasing the performance of heating systems in public transport vehicles.
\end{abstract}


Keywords: Heating systems, Public transport, Control.

\section{Referencias}

[1] Al Faruque, M. A., Vatanparvar, K., (2016) "Modeling, analysis, and optimization of Electric Vehicle HVAC systems", Proceedings of the Asia and South Pacific Design Automation Conference, ASP-DAC, vol. 25-28Janu, pp. 423-428.

[2] Bellocchi, S., Leo Guizzi, G., Manno, M., Salvatori, M., Zaccagnini, A., (2018) "Reversible heat pump HVAC system with regenerative heat exchanger for electric vehicles: Analysis of its impact on driving range", Applied Thermal Engineering, vol. 129, pp. 290-305.

[3] Farzaneh, Y., Tootoonchi, A. A., (2008) "Controlling automobile thermal comfort using optimized fuzzy controller", Applied Thermal Engineering, vol. 28, pp. 1906-1917.

[4] He, H., Yan, M., Sun, C., Peng, J., Li, M., Jia, H., (2018) "Predictive air-conditioner control for electric buses with passenger amount variation forecast $25 \%$, Applied Energy, vol. 227, pp. 249-261.

[5] Marcos, D., Pino, F. J., Bordons, C., Guerra, J. J., (2014) "The development and validation of a thermal model for the cabin of a vehicle", Applied Thermal Engineering, vol. 66, pp. 646-656.

[6] Pala, U., Oz, H. R., (2015) "An investigation of thermal comfort inside a bus during heating period within a climatic chamber", Applied Ergonomics, vol. 48, pp. 164-176.

[7] Ruz, M., Garrido, Vázquez, F. Morilla, F. (2017) "A hybrid modeling approach for steady-state optimal operation of vapor compression refrigeration cycles", Applied Thermal Engineering, vol. 120, pp. 74-87.

[8] Shoukat Choudhury, M. A. A., Thornhill, N. F., Shah, S. L., (2005) "Modelling valve stiction", Control Engineering Practice, vol. 13, pp. 641-658.

[9] Torregrosa-Jaime, B., Bjurling, F., Corberán, J. M., Di Sciullo, F., Payá, J., (2015) “Transient thermal model of a vehicle's cabin validated under variable ambient conditions", Applied Thermal Engineering, vol. 75, pp. 45-53.

[10] Zhi, Z., Peng, D., Pei, Z., (2013) "Performance analysis and simulation of automotive air conditioning systems", Applied Mechanics and Materials, vol. 261-262, pp. 357-361.

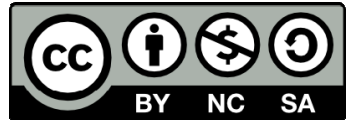

(C) 2021 by the authors. Submitted for possible open access publication under the terms and conditions of the Creative Commons Attribution CC BY-NC-SA 4.0 license (https://creativecommons.org/licenses/byncsa/4.0/deed.es). 